\title{
miRNA-122: Can it unravel an alternative pathway in early diagnosis and prognosis of type 2 diabetes mellitus?
}

Dimitrios Ioannis Patoulias ${ }^{1,2}$

${ }^{1}$ Second Propedeutic Department of Internal Medicine, Aristotle University
of Thessaloniki, General Hospital Hippokration, Thessaloniki, Greece
2Department of Internal Medicine, General Hospital of Veria, Veria, Greece

Submitted: 27 April 2018

Accepted: 1 June 2018

Arch Med Sci Atheroscler Dis 2018; 3: e68-e71

DOI: https://doi.org/10.5114/amsad.2018.76823

Copyright @ 2018 Termedia \& Banach

MicroRNAs (miRNAs) have recently emerged as an attractive and developing cluster of biomarkers for several diseases, with ongoing experimental and human studies assessing their potential diagnostic and prognostic utility in clinical practice. There is also increasing evidence that they may serve as potential therapeutic targets.

Type 2 diabetes mellitus (T2DM) is a chronic disease, constituting a major cause of morbidity and mortality worldwide. The outlook is rather pessimistic, as its prevalence is expected to increase significantly over the coming decades. Thus, there is an undeniable need for biomarkers which will permit the early identification of patients being at high risk for the manifestation of T2DM and their monitoring, after establishing the diagnosis and initiating antidiabetic treatment. The latter may be a major preventive measure, considering the development of diabetic microvascular and macrovascular complications.

The pathophysiology of T2DM is multifactorial. One major mechanism is ectopic lipid accumulation in the liver, which inhibits insulin signaling and promotes hepatic insulin resistance [1]. Additionally, prolonged hyperlipemia has been associated with progressive $\beta$-cell dysfunction and $\beta$-cell apoptosis, resulting in impaired insulin production [2].

miRNA-122 is the most abundant miRNA in the liver, accounting for almost $75 \%$ of total miRNA expression in this organ [3]. It has been implicated in regulation of lipid homeostasis, free fatty acid (FFA) metabolism and lipogenesis [4]. More specifically, it is crucial for cholesterol biosynthesis and very low-density lipoprotein (VLDL) secretion in the liver [3]. A direct association between miRNA-122 and hepatocyte nuclear factor $4 \alpha(\mathrm{HNF}-4 \alpha)$ has been recently described, leading finally to abnormal cholesterol homeostasis and increased FFA and triglyceride levels [5]. The experimental study by Yang et al. showed that overexpression of c-Jun $\mathrm{N}$-terminal kinase 1 (JNK1) induces downregulation of miRNA-122 and induction of protein tyrosine phosphatase 1B (PTP1B). The latter process leads to inactivating phosphorylation of $\mathrm{HNF}-4 \alpha$, finally resulting in hepatic insulin resistance. According to the researchers, development and administration of JNK1 inhibitors can increase miRNA-122 expression, leading to amelioration of insulin resistance and overall improvement in metabolic profile [6].

There is increasing evidence, deriving from both human and experimental studies, that miRNA-122 is associated with obesity and related metabolic disorders, including insulin resistance, impaired glucose tolerance and finally development of T2DM [7-14].

\author{
Corresponding author: \\ Dimitrios loannis Patoulias \\ Department of \\ Internal Medicine \\ General Hospital \\ of Veria \\ 3 B M. Alexandrou St \\ 57010 Thessaloniki, Greece \\ Phone: +30 6946900777 \\ E-mail: dipatoulias@gmail.com
}


In their pilot study, Delic et al. attempted to identify miRNA changes during the progression of T2DM in Zucker diabetic fatty (ZDF) rats [7]. The researchers distinguished the following disease stages: a) pre-diabetes ( 6 weeks), b) hyperinsulinemia (8 weeks), c) $\beta$-cell failure (11 weeks) and d) late stage diabetes (17 weeks). They observed that the circulating levels of miRNA-122 were significantly increased when $\beta$-cell failure occurred (7-fold elevation). However, besides the direct relationship with $\beta$-cell failure, the significant increase in miRNA-122 levels also correlated with the fatty liver that ZDF rats developed during the disease course [7]

In another study, Jones et al. recruited initially obese patients undergoing gastric bypass surgery, who were further classified as patients with T2DM or patients without T2DM, either with insulin sensitivity (IS) or insulin resistance (IR), along with normal weight non-diabetic controls [8]. The researchers observed a strong positive correlation of circulating miRNA-122 levels with IR ( $p<0.05)$. They also attempted to identify miRNA changes in a murine model of obesity, by feeding male C567BL/6 mice either normal chow or a high-fat, high-sucrose (HFHS) diet. Plasma miRNA-122 levels were significantly elevated in HFHS fed mice, compared to those fed normal chow $(p=0.008)$. The researchers also observed a significant increase in miRNA-122 levels in subcutaneous fat in HFHS mice, compared to controls $(p=0.04)$. Careful interpretation of these results leads to the conclusion that circulating miRNA-122 may serve as a biomarker for obesity and metabolic syndrome related comorbidities [8].

Wang et al. performed one of the largest relevant human studies, stratifying 112 obese and control subjects, while 230 participants were recruited for the validation study [9]. They observed that obese participants exhibited 3.07-fold higher circulating miRNA-122 levels, compared with normal weight controls $(p<0.0001)$, while miRNA-122 levels correlated significantly with alanine aminotransferase, triglycerides, high-density lipoprotein $(\mathrm{HDL})$ cholesterol, and more interestingly, with body mass index (BMI) and HOMA-IR $(p<$ 0.01). After adjustment for age, gender, obesity, blood pressure, lipid profile, and liver function, circulating miRNA-122 positively correlated with increased odds for insulin resistance $(O R=3.379$ per each SD unit increase in miRNA-122 levels, $95 \% \mathrm{Cl}: 1.141-10.007, p=0.028)$. These findings are highly suggestive of a potential role of miRNA-122 as a biomarker for obesity and IR [9].

In another study with similar design conducted by Ortega et al. it was found that circulating miRNA-122 levels were increased in obese patients (30 $\leq \mathrm{BMI}<40 \mathrm{~kg} / \mathrm{m}^{2}$ ), compared to non-obese patients [10]. However, it was also observed that plasma miRNA-122 concentration was decreased in morbidly obese patients (BMI $\geq 40 \mathrm{~kg} / \mathrm{m}^{2}$ ). The researchers observed a marked decrease in circulating miRNA-122 levels, after surgically induced weight loss through Roux-en-Y gastric bypass (RYGB), as well. No significant association was documented in those patients that underwent diet-induced weight loss [10].

Regarding childhood obesity, Prats-Puig et al. attempted through a cross-sectional validation study followed by a longitudinal validation study to identify those circulating miRNAs most relevant to obesity [11]. They observed that miRNA-122 levels were significantly higher in the obese children group, compared to the lean subjects ( $p=$ 0.017). Indeed, circulating miRNA-122 levels correlated positively with both clinical (BMI, fat mass, waist perimeter) and biochemical obesity-associated parameters (HOMA-IR, circulating lipids, high-molecular-weight adiponectin) [11].

Wu et al. attempted to shed light on the underlying pathophysiologic mechanisms triggered after RYGB in an animal model using Sprague Dawley rats [12]. The researchers observed that circulating miRNA-122 levels were significantly downregulated in RYGB-operated animals, exhibiting a 56-fold decrease $(p=0.0095)$. They also observed an imbalance in reduction between circulating and hepatic miRNA-122 (99\% vs. 30\%, respectively). Their further metabolic and miRNA-122 target expression analysis revealed that miRNA-122 contributes to the control of energy metabolism, implicating increased glucose transportation, glycolysis, tricarboxylic acid cycle, pentose phosphate shunt and fatty oxidation, along with decreased gluconeogenesis and ketogenesis. The latter are suggestive of significant homeostatic activity of miRNA-122 in obesity and closely related co-morbidities, mainly T2DM [12].

De Candia et al. aimed to identify a plasma miRNA profile, in order to detect those patients exhibiting glucose dysmetabolism, although not yet diagnosed with T2DM [13]. Significantly, they observed that miRNA-122 levels were elevated in patients with impaired glucose tolerance (IGT), compared to those with normal glucose tolerance (NGT), attributing potential early diagnostic value to the discussed biomarker. The most accentuated miRNA-122 dysregulation was observed in non-progressor (NP) patients with IGT. The direct comparison of plasma miRNA-122 levels between NP and progressor (P) IGT patients was non-significant. The authors concluded that the increased levels of miRNA-122 in NP IGT patients may reveal the potential regulatory role of this miRNA in metabolic balance [13].

The study recently published by Willeit et al. is a hallmark in the field [14]. The researchers observed that patients with metabolic syndrome or 
T2DM had significantly higher levels of circulating miRNA-122, compared to controls ( $p<0.001$ for both comparisons). There was also a significant positive correlation with elevated liver enzymes, measures of adiposity (BMI and waist-to-hip ratio), logHOMA-IR, high-sensitivity C-reactive protein (hsCRP), triglycerides and HDL-C ( $p<0.001$ for all). More interestingly, after a 15-year follow-up period in the Bruneck study, the researchers observed that multivariable adjusted risk ratios per one-SD higher log miR-122 were $1.60(95 \% \mathrm{Cl}$ : 1.30-1.96; $p<0.001)$ for metabolic syndrome and $1.37(1.03-1.82 ; p=0.021)$ for T2DM. As the authors mention in their discussion, "elevated serum levels of miRNA-122 antedate the manifestation of metabolic syndrome and type 2 diabetes". Thus, it can be deduced that miRNA-122 is a novel biomarker reflecting insulin resistance, with potential applicability in the early detection of highrisk patients and their monitoring after diagnosis of T2DM.

Of course, certain considerations regarding the clinical utility of miRNAs, including miRNA-122, as disease biomarkers emerge. The biodistribution of circulating miRNAs and their potential functional role at the tissue level and on target cells has be to further elucidated [15]. Effects of miRNA-122 on multiple pathways have to be addressed, as it regulates the expression of more than 170 interacting genes, in different tissues and cells. Thus, its specificity is still under investigation [16].

In addition, the processing procedure of the biomolecules from body fluids or tissue specimens, their storage conditions and the sample preparation have to be optimized, along with the development of common protocols among the different laboratories, as even small amendments in the whole process can significantly alter miRNA levels in the final sample [17].

Besides the undeniable need for a standardized, common protocol for both collection and analysis of biological samples, further concerns emerge. Barutta et al. question whether circulating miRNAs derived from damaged/necrotic cells constitute a potential confounder and if measurement of miRNAs enclosed in microvesicles or exosomes is preferable [18]. They also express their concerns regarding the effect of environmental variables on the levels of circulating miRNAs, and whether those variables can overlap the underlying pathophysiological mechanisms of the disease of interest [18].

Overall, miRNAs may function as diagnostic and prognostic biomarkers obtained from minimally invasive techniques, making them highly attractive in clinical practice. However, circulating miRNA profiling still has potential for significant technological progress and appropriate standardization [19]. Development of affordable, point-of- care miRNA diagnostic tests, along with establishment of their prognostic value in large prospective studies in longitudinal cohorts, may constitute a real revolution in the field of diagnosis and prognosis of T2DM [18, 20].

In conclusion, as insulin resistance is a core defect in T2DM, the investigation of novel biomarkers that enable the quantification of this pathophysiological mechanism may be crucial, for the prevention of both the manifestation and progression of the disease. miRNA-122 seems to be a highly valuable biomarker. It can also be integrated in novel diagnostic panels, along with well-established diagnostic and prognostic markers of T2DM (such as $\mathrm{HbA}_{1 \mathrm{c}}$, FPG, PPG and HOMA-IR) or even with other related miRNAs, in order to significantly increase its diagnostic and prognostic value. As Farr and colleagues state, "validation and refinement of a miRNA biomarker signature for diabetes would allow identification and intervention of individuals at risk of this disease, as well as stratification and monitoring of patients with established diabetes" [21].

Further large prospective studies are needed in order to elucidate this hypothesis. However, miRNA-122 promises to unravel new pathways in the diagnosis and prognosis of T2DM.

\section{Conflict of interest}

The author declares no conflict of interest.

\section{References}

1. Shulman GI. Ectopic fat in insulin resistance, dyslipidemia, and cardiometabolic disease. N Engl J Med 2014; 371: 2237-8.

2. Leibowitz G, Kaiser N, Cerasi E. Beta-cell failure in type 2 diabetes. J Diabetes Investig 2011; 2: 82-91.

3. Fernández-Hernando C, Ramírez CM, Goedeke L, Suárez Y. MicroRNAs in metabolic disease. Arterioscler Thromb Vasc Biol 2013; 33: 178-85.

4. Fernández-Hernando C, Suárez Y, Rayner KJ, Moore KJ. MicroRNAs in lipid metabolism. Curr Opin Lipidol 2011; 22: 86-92.

5. De Rosa S, Arcidiacono B, Chiefari E, Brunetti A, Indolfi C, Foti DP. Type 2 diabetes mellitus and cardiovascular disease: genetic and epigenetic links. Front Endocrinol (Lausanne) 2018; 9: 2.

6. Yang YM, Seo SY, Kim TH, Kim SG. Decrease of microRNA-122 causes hepatic insulin resistance by inducing protein tyrosine phosphatase $1 \mathrm{~B}$, which is reversed by licorice flavonoid. Hepatology 2012; 56: 2209-20.

7. Delic D, Eisele C, Schmid R, Luippold G, Mayoux E, Grempler R. Characterization of micro-RNA changes during the progression of type 2 diabetes in Zucker diabetic fatty rats. Int I Mol Sci 2016; 17: E665.

8. Jones A, Danielson KM, Benton MC, et al. miRNA signatures of insulin resistance in obesity. Obesity 2017; 25: 1734-44.

9. Wang R, Hong J, Cao Y et al. Elevated circulating microRNA-122 is associated with obesity and insulin resistance in young adults. Eur J Endocrinol 2015; 172: 291-300. 
10. Ortega FJ, Mercader JM, Catalan V, et al. Targeting the circulating microRNA signature of obesity. Clin Chem 2013; 59: 781-92.

11. Prats-Puig A, Ortega FJ, Mercader JM, et al. Changes in circulating microRNAs are associated with childhood obesity. J Clin Endocrinol Metab 2013; 98: E1655-60.

12. Wu Q, Li JV, Seyfried F, et al. Metabolic phenotype-microRNA data fusion analysis of the systemic consequences of Roux-en-Y gastric bypass surgery. Int J Obes (Lond) 2015; 39: 1126-34.

13. de Candia P, Spinetti G, Specchia C, et al. A unique plasma microRNA profile defines type 2 diabetes progression. PLoS One 2017; 12: e0188980.

14. Willeit P, Skroblin P, Moschen AR, et al. Circulating microRNA-122 is associated with the risk of new-onset metabolic syndrome and type 2 diabetes. Diabetes 2017; 66: 347-57.

15. Szabo G, Bala S. MicroRNAs in liver disease. Nat Rev Gastroenterol Hepatol 2013; 10: 542-52.

16. Pirola CJ, Gianotti TF, Castaño GO, Sookoian S. Circulating microRNA-122 signature in nonalcoholic fatty liver disease and cardiovascular disease: a new endocrine system in metabolic syndrome. Hepatology 2013; 57: 2545-7.

17. Vaishya S, Sarwade RD, Seshadri V. MicroRNA, proteins, and metabolites as novel biomarkers for prediabetes, diabetes, and related complications. Front Endocrinol 2018; 9: 180.

18. Barutta F, Bellini S, Mastrocola R, Bruno G, Gruden G. MicroRNA and microvascular complications of diabetes. Int J Endocrinol 2018; 2018: 6890501.

19. Moldovan L, Batte KE, Trgovcich J, Wisler J, Marsh CB, Piper M. Methodological challenges in utilizing miRNAs as circulating biomarkers. J Cell Mol Med 2014; 18: 371-90.

20. Pritchard CC, Cheng HH, Tewari M. MicroRNA profiling: approaches and considerations. Nat Rev Genet 2012; 13: 358-69.

21. Farr RJ, Joglekar MV, Hardikar AA. Circulating microRNAs in diabetes progression: discovery, validation, and research translation. EXS 2015; 106: 215-44. 